\title{
Experimental observation of the avoided crossing of two $S$-matrix resonance poles in an ultracold atom collider
}

\author{
Matthew Chilcott $\odot,{ }^{1}$ Ryan Thomas $\odot,{ }^{1,2}$ and Niels Kjærgaard $\odot^{1, *}$ \\ ${ }^{1}$ Department of Physics, QSO-Quantum Science Otago, and Dodd-Walls Centre for Photonic and Quantum Technologies, \\ University of Otago, Dunedin 9016, New Zealand \\ ${ }^{2}$ Department of Quantum Science, Research School of Physics, The Australian National University, Canberra 2601, Australia
}

(Received 4 November 2020; accepted 6 August 2021; published 2 September 2021)

\begin{abstract}
In quantum mechanics, collisions between two particles are captured by a scattering matrix which describes the transfer from an initial entrance state to an outgoing final state. Analyticity of the elements of this $S$ matrix enables their continuation onto the complex energy plane and opens up a powerful and widely used framework in scattering theory, where bound states and scattering resonances for a physical system are ascribed to $S$-matrix poles. In the Gedankenexperiment of gradually changing the potential parameters of the system, the complex energy poles will begin to move, and in their ensuing flow, two poles approaching will interact. An actual observation of this intriguing interaction between scattering poles in a collision experiment has, however, been elusive. Here, we expose the interplay between two scattering poles relating to a shape resonance and a magnetically tunable Feshbach resonance by studying ultracold atoms with a laser-based collider. We exploit the tunability of the Feshbach resonance to observe a compelling avoided crossing of the poles in their energies which is the hallmark of a strongly coupled system.
\end{abstract}

DOI: 10.1103/PhysRevResearch.3.033209

\section{INTRODUCTION}

Atomic collision resonances occur when a scattering state in the energy continuum couples to a quasibound state of the interatomic potential. Near a resonant energy, the scattering of colliding atoms may be dramatically affected and either enhanced or suppressed. According to the superposition principle of quantum mechanics, the final state of the system results from a linear operator $\hat{S}$ acting on the initial state of the incoming particles [1]. The $S$ matrix responsible for this transformation is a function of the center-of-mass collision energy $E$, which is a positive, real quantity for a physical process. It may, however, be analytically continued into the complex plane for most physical interaction potentials. Remarkably, poles of this analytically extended $S$ matrix occurring at nonphysical, complex values of $E$ are intimately related to the scattering resonances observed when scanning the collision energy along the real energy axis [2-4]. Indeed, characterizing a resonance through its pole position provides a powerful alternative to Breit-Wigner parametrization [5,6].

The locations of $S$-matrix poles are determined by the interaction potential of the system Hamiltonian. As a consequence, upon changing the parameters describing the potential the poles will move and their ensuing flow has been investigated

\footnotetext{
*niels.kjaergaard@ otago.ac.nz

Published by the American Physical Society under the terms of the Creative Commons Attribution 4.0 International license. Further distribution of this work must maintain attribution to the author(s) and the published article's title, journal citation, and DOI.
}

theoretically, for example, in the cases of various potential wells [7-12] or coupled two-state systems [13-17]. In the latter instance it was shown that interchannel coupling may give rise to avoided crossings of poles similarly to the LandauZener avoided crossing for coupled discrete states of a bound system. An observation of this intriguing interplay between poles of the $S$ matrix in a collision experiment has, however, been lacking.

In this study, we experimentally investigate atomic scattering when both a shape resonance and a Feshbach resonance are at work at the same time. In particular, we observe the signature of an avoided crossing between the two $S$-matrix poles corresponding to the resonances. We consider rubidium atoms colliding at energies in the nano-eV range, with one pole originating from a $d$-wave shape resonance and the other from a $d$-wave magnetic Feshbach resonance. Our system is well described by a two-channel picture and illuminates with remarkable clarity the interplay between the two archetypal types of resonances found in atomic scattering: shape and Feshbach resonances. Because both resonances have a $d$-wave character, the interplay dramatically affects the scattering cross section at the nominal position of the shape resonance while scattering at threshold remains $s$ wave and largely unaffected.

\section{SCATTERING RESONANCES}

In atomic scattering, shape resonances are typically formed by coupling to a quasibound state accommodated behind the centrifugal barrier for collisions with nonzero angular momentum. Atomic shape resonances have been observed using a number of techniques including photoassociation 
(a)

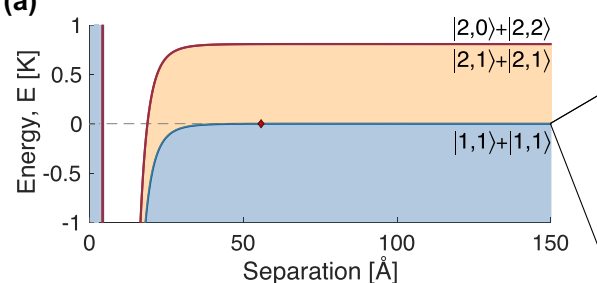

(c)

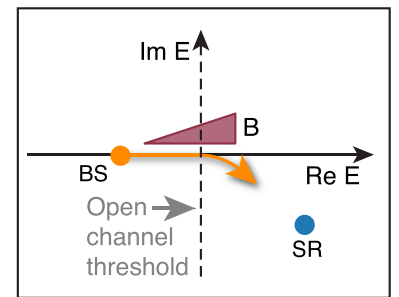

(b)

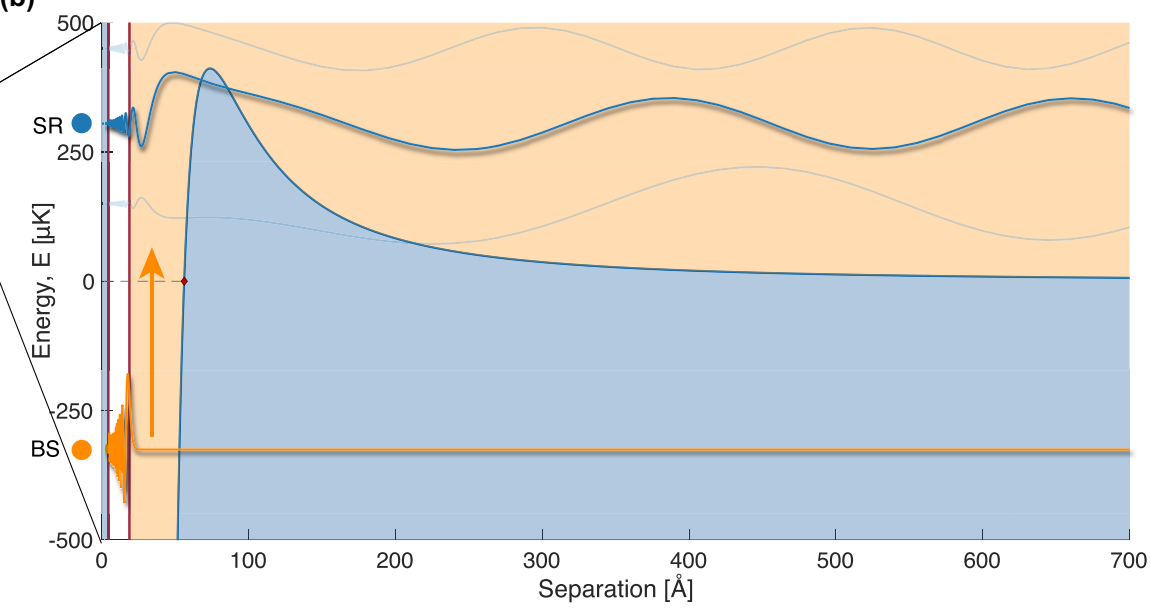

FIG. 1. Scenario for atomic scattering with two $S$-matrix poles simultaneously in play. (a) Relevant atomic-basis potentials for cold $d$-wave collisions between two ${ }^{87} \mathrm{Rb}$ atoms in the $\left|F=1, m_{F}=1\right\rangle$ hyperfine state [25]. The difference between the $|2,0\rangle+|2,2\rangle$ and $|2,1\rangle+|2,1\rangle$ potentials is not discernible for the window shown. The energy scale also renders the $\ell=2$ centrifugal barriers invisible, but the red diamond shows the classical turning point inside the barrier for the $|1,1\rangle+|1,1\rangle$ channel at threshold (dashed line). (b) Scattering state wave functions in the $|1,1\rangle+|1,1\rangle$ entrance channel [blue, with dark blue highlighting a $d$-wave shape resonance (SR)]. For an external magnetic field $B=927.5 \mathrm{G}$ a bound state (BS) resides below threshold of the open channel: the orange wave function shows its $|2,0\rangle+|2,2\rangle$ component; the other significant component (not shown), $|2,1\rangle+|2,1\rangle$, is very similar. Upon increasing the external magnetic field, the position of the bound state moves up in energy as indicated by the arrow. It eventually crosses the $|1,1\rangle+|1,1\rangle$ threshold to become a quasibound state corresponding to a Feshbach resonance in this channel. (c) The bound state and the shape resonance correspond to $S$-matrix poles on the complex energy plane and the magnetic field will tune their relative positions.

spectroscopy [18], molecule loss spectroscopy [19], molecular dissociation [20], or in colliderlike settings [21-24]. Residing in the open channel the quasibound state, and hence the resonance, typically only has minuscule tuning with external fields [19].

In contrast to shape resonances, Feshbach resonances are highly tunable. These occur due to the presence of a bound state in an energetically closed channel which has a different spin to the entrance channel. With interchannel coupling present one can use the Feshbach resonance to vary the entrance scattering length over a wide range by tuning the resonance position via optical or magnetic fields. Manipulating interactions in this way is an indispensable tool in ultracold atomic physics and paved the way for landmark quantum many-body experiments such as the formation of superfluid solitons [28], the realization of the BEC-BCS crossover [29,30], and self-bound quantum droplets [31].

\section{SYSTEM UNDER STUDY}

Figures 1(a)-1(c) present a scenario where a shape resonance and a magnetic Feshbach resonance are simultaneously in play. The shape resonance is associated with open-channel scattering-state wave functions (blue) and manifests as an amplitude increase (dark blue) behind the centrifugal barrier at a particular energy [see Fig. 1(b)]. The Feshbach resonance, on the other hand, relates to a bound state with a wave function (orange) which decays rapidly with atomic separation. In Fig. 1(b) this state is depicted to lie below the open channel threshold at $E=0$. For a magnetic Feshbach resonance as we explore here, the closed channel hosting the bound state has a different magnetic moment than the open channel state. In this two-channel setting, Fig. 1(c) shows the poles on the so-called $L(-+)$ Riemann sheet [32,33] of the analytically continued $S$ matrix. If coupling between the channels is introduced, a bound state in the closed channel with $E>0$ becomes an "unstable bound state" [32] that can decay into the open channel by a change in spin state. By adjusting an external magnetic field, the offset between the open and closed channels can be varied as indicated by the arrow in Fig. 1(b). As the bound state moves to a positive energy, coupling to the open channel makes it unstable and it becomes the origin of a collisional resonance while its corresponding $S$-matrix pole moves off the real energy axis in Fig. 1(c).

A theoretical description of the interaction between a shape resonance and Feshbach resonance has been established by Dür et al. [34], who provided a framework for interpreting experiments on the dissociation of rubidium Feshbach molecules into multiple partial waves. In particular, a $d$-wave shape resonance made a compelling spatial imprint on the halo of dissociating molecules [20]. As the starting point of these experiments was a true bound molecule, the explanation required the introduction of the concept of "half collisions" as the dissociation experiment did not include the incoming stage of a collision. In contrast, we investigate the interplay between a Feshbach resonance and a shape resonance in a genuine collision experiment and seek an interpretation in terms of an avoided crossing of scattering poles. Along with a $d$-wave shape resonance at $E / k_{B} \sim 300 \mu \mathrm{K}$, we make use of a narrow $d$-wave Feshbach resonance near $930 \mathrm{G}$ for ultracold ${ }^{87} \mathrm{Rb}$ in the $\left|F, m_{F}\right\rangle \equiv|1,1\rangle$ hyperfine state [35]. This Feshbach resonance relates to a closed channel $\ell=2$ 
(a)
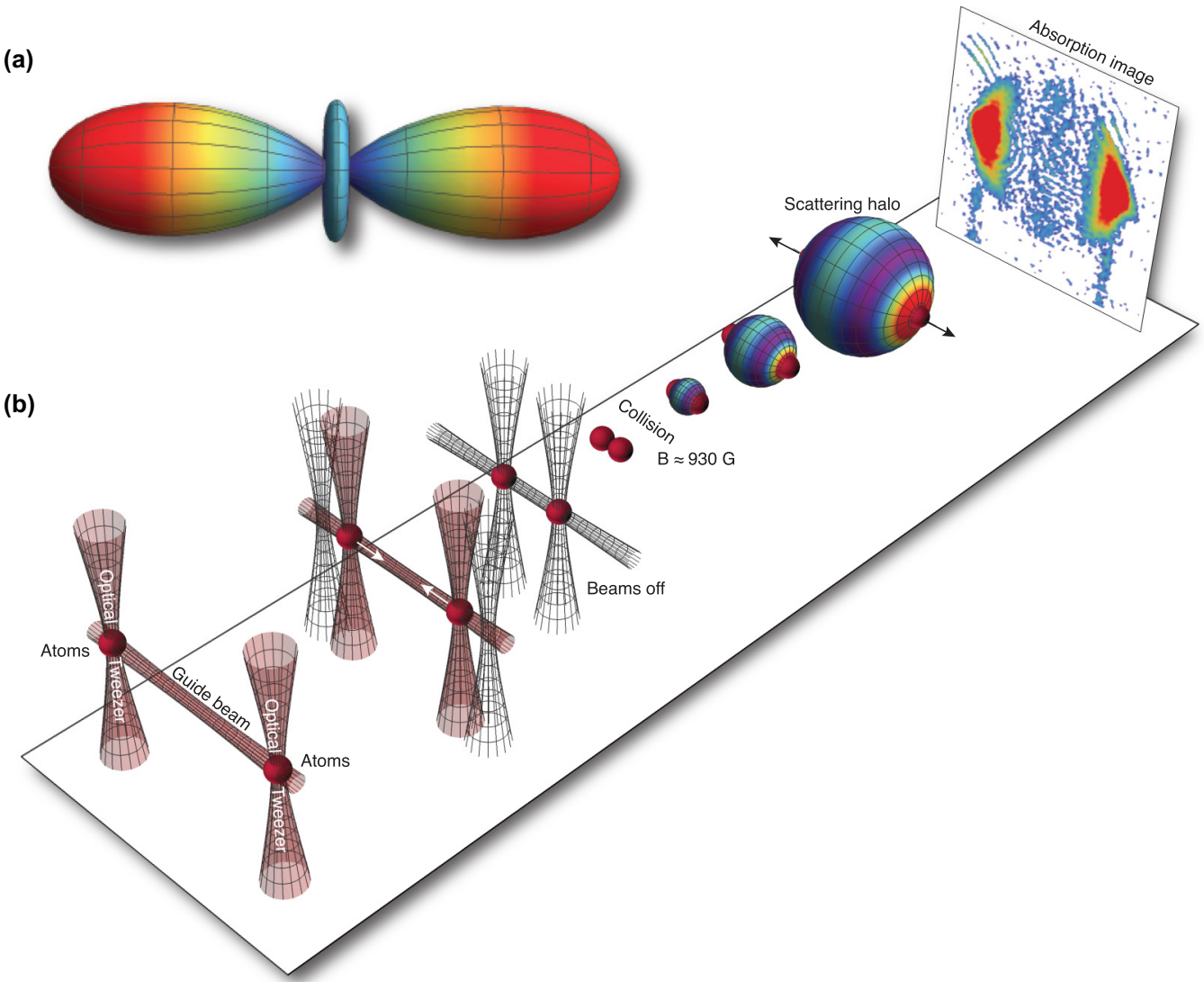

FIG. 2. $d$-wave scattering in our optical collider. (a) $d$-wave angular emission pattern which dominates the distribution of elastically scattered atoms in the collider experiment. (b) Optical collider procedure. Atomic clouds confined by laser beams are steered to collide in free space while subject to a magnetic field. The resulting $d$-wave dominated scattering halo is detected using absorption imaging.

molecular state designated by the quantum numbers $\left(F_{1}=\right.$ $\left.2, F_{2}=2\right) v^{\prime}=-5, M=2$, where $M=m_{F_{1}}+m_{F_{2}}$ and $v^{\prime}$ is the vibrational quantum number counting from the $\left(F_{1}=\right.$ $2, F_{2}=2$ ) threshold. Represented in the separated atomic basis, the molecular state decomposes predominantly into $|2,0\rangle+|2,2\rangle$ and $|2,1\rangle+|2,1\rangle$ (cf. Fig. 1 and [25]).

\section{EXPERIMENT}

\section{A. Procedure}

Our experimental setup has been described previously [36]. In brief, we begin our collider experiment with a cloud of ${ }^{87} \mathrm{Rb}$ atoms in the $|2,2\rangle$ state held in an optical dipole trap. The dipole trap is formed by a crossed pair of red-detuned laser beams-a static horizontal beam defines the collision axis, while a vertical beam is steered by a pair of crossed acoustooptic deflectors [37]. By rapidly toggling the frequency of the deflector $\mathrm{rf}$ drive, we produce a pair of time-averaged optical traps, which we pull apart along the horizontal beam forming two clouds separated by $0.5 \mathrm{~mm}$. We then transfer the cloud to the $|1,1\rangle$ state by adiabatic rapid passage [38] using a swept microwave field in the presence of a small magnetic bias field. The clouds are evaporatively cooled to a temperature of $\sim 800 \mathrm{nK}$ by lowering the power of the horizontal trap beam. To minimize heating of the clouds at our higher collision energies, the clouds are then further separated up to $2 \mathrm{~mm}$, providing a longer runup and reducing the magnitude of the acceleration needed.
Once two separated clouds have been prepared, we apply a magnetic field along the collision axis using a pair of watercooled coils in the Helmholtz configuration, which carries a current regulated to the sub-ppm level [39]. The field strength is selected to inspect the Feshbach resonance and the vertical trapping beams are steered to accelerate the atomic clouds towards each other as illustrated in Fig. 2. All trapping beams are turned off before the collision and the clouds collide in free space in the presence of the field from the Helmholtz coils. Following the collision, we perform absorption imaging of the scattering halo which projects the atomic density distribution onto a plane [Fig. 2(b)]. We integrate the acquired absorption image perpendicular to the collision axis and fit a model based on the projections of both the unscattered clouds and the relevant scattered partial-wave components. The curve fit extracts the fraction of scattered atoms, $\mathcal{S}$, which can be related to the scattering cross section $\sigma$ by

$$
\mathcal{S}(B, E)=\frac{\alpha(E) \sigma(B, E)}{1+\alpha(E) \sigma(B, E)} .
$$

Here, $\alpha$ is a fitted parameter in our model whose value depends on the density and cross-sectional geometry of the cloud [36].

\section{B. Measurements}

Figure 3 shows our measurements of $\mathcal{S}(B, E)$ over a domain spanning magnetic fields from 900 to $960 \mathrm{G}$ and 


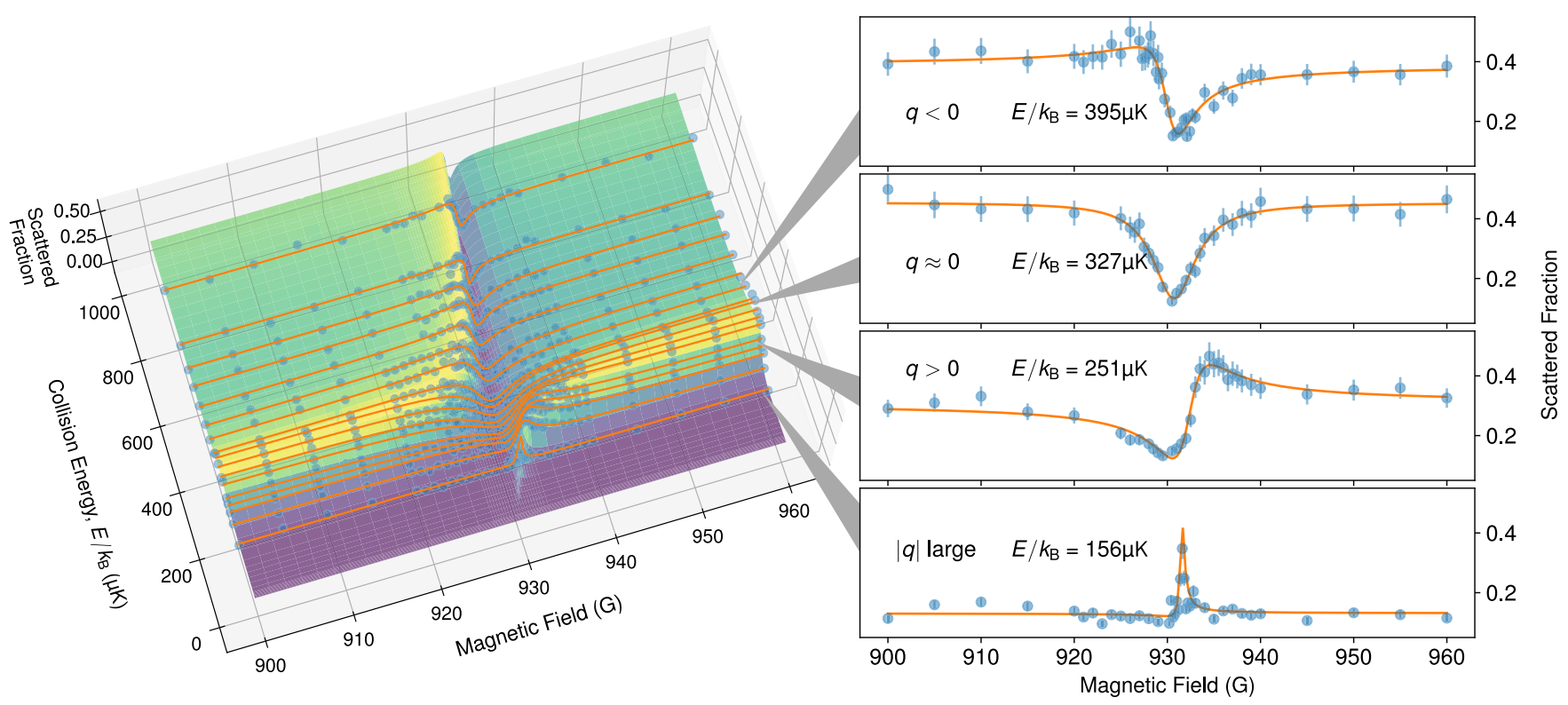

FIG. 3. Fraction of scattered atoms as a function of both magnetic field and collision energy. Experimentally acquired data are shown as blue dots while the orange lines are curve fits of Beutler-Fano lines shapes given by Eq. (2). The surface is an estimate of the scattered fraction based on coupled-channels predictions of the elastic cross section. Insets: Fano profiles at selected collision energies spanning the shape resonance, with error bars showing the standard deviation.

collision energies ranging $150-850 \mu \mathrm{K}$. We also present predictions based on coupled-channels calculations of the elastic cross section (See Appendix A) which displays excellent agreement with our experimental measurements. In the experiment, the scattered fractions were acquired by fixing $E$ while scanning $B$, and insets in Fig. 3 show four exemplary $B$-field scans which capture resonance features that are all well described by a Beutler-Fano line shape [40]. As the energy passes over the nominal shape resonance position $(\sim 300 \mu \mathrm{K})$, we observe a handedness flip of the Fano profile (see insets of Fig. 3). Additionally, we can see that the profile broadens for scans around $300 \mu \mathrm{K}$ as a direct result of the increase in the open-channel short-range wave-function amplitude [see Fig. 1(b)] enhancing the coupling of incoming particles to the bound state in the closed channel.

\section{Beutler-Fano lines}

The Beutler-Fano line shape which describes the nearresonance $d$-wave scattering is a function of magnetic field $B$ and collision energy $E$. When combined with the cross section from other partial waves, $\sigma_{\ell \neq 2}$, we have

$$
\begin{aligned}
\sigma(E, B)= & \underbrace{\frac{4 \pi \hbar^{2}(2 \ell+1)}{m E}\left\{\frac{[\epsilon(E, B)+q]^{2}}{\left(1+q^{2}\right)\left[1+\epsilon(E, B)^{2}\right]}\right\}}_{\sigma_{\ell=2}} \\
& +\sigma_{\ell \neq 2}(E),
\end{aligned}
$$

with the dimensionless parameter $\epsilon(E, B)=2(B-$ $\left.B_{0}\right) / \Gamma(E)$, where $B_{0}$ and $\Gamma$ are the position and width of the resonance respectively. The parameter $q$ defines the shape of the Fano profile and changes sign as the profile flips in Fig. 3-a phenomenon known as $q$ reversal [34]. We fit each data scan to a model which combines Eqs. (1) and (2) and plot the fitted curves in Fig. 3. From the fits we extract the cross section at a slice of the magnetic field at constant energy, along with the shape parameter $q$ and width $\Gamma$.

\section{INTERACTING S-MATRIX POLES}

To interpret our experiment within the framework of $S$-matrix scattering poles, we analytically continue the $S$ matrix provided by our coupled-channels calculations into the complex energy plane by fitting a Padé approximant (See Appendix B).

In Fig. 4(a), we plot the trajectory of the poles as they move in the complex plane with an increasing magnetic field (see also Movie 1 in the Supplemental Material [41]). The position of a pole, $z=E-\frac{i}{2} \gamma$, provides the energy $E$ and width $\gamma$ of a resonance experienced along the real energy line. The pole following the blue curve is initially the source of the shape resonance, while the pole following the orange curve, initially residing well below threshold, is associated with the bound state in the closed channel, that is, the budding Feshbach resonance. As we increase the magnetic field, both poles move to higher (real) energies, and eventually the orange pole takes over the position of the shape resonance, while the blue pole continues tuning as the Feshbach resonance. In the intermediate region where the two resonances interact, the role of each pole is not clearly defined.

Inset above the pole trajectories in Fig. 4(a), we show the magnetic resonance as witnessed at three fixed energies-our collected data - and can observe which poles contribute to the form of the resonance. In particular, we observe that at energies well below the nominal position of the shape resonance $(\sim 300 \mu \mathrm{K})$, the movement and presence of the orange pole is responsible for the profile in magnetic field. At energies above $300 \mu \mathrm{K}$, it is the blue pole moving past that makes an imprint on the scattered fraction. Close to $300 \mu \mathrm{K}$, both poles are involved, with their interference resulting in a dip profile. 


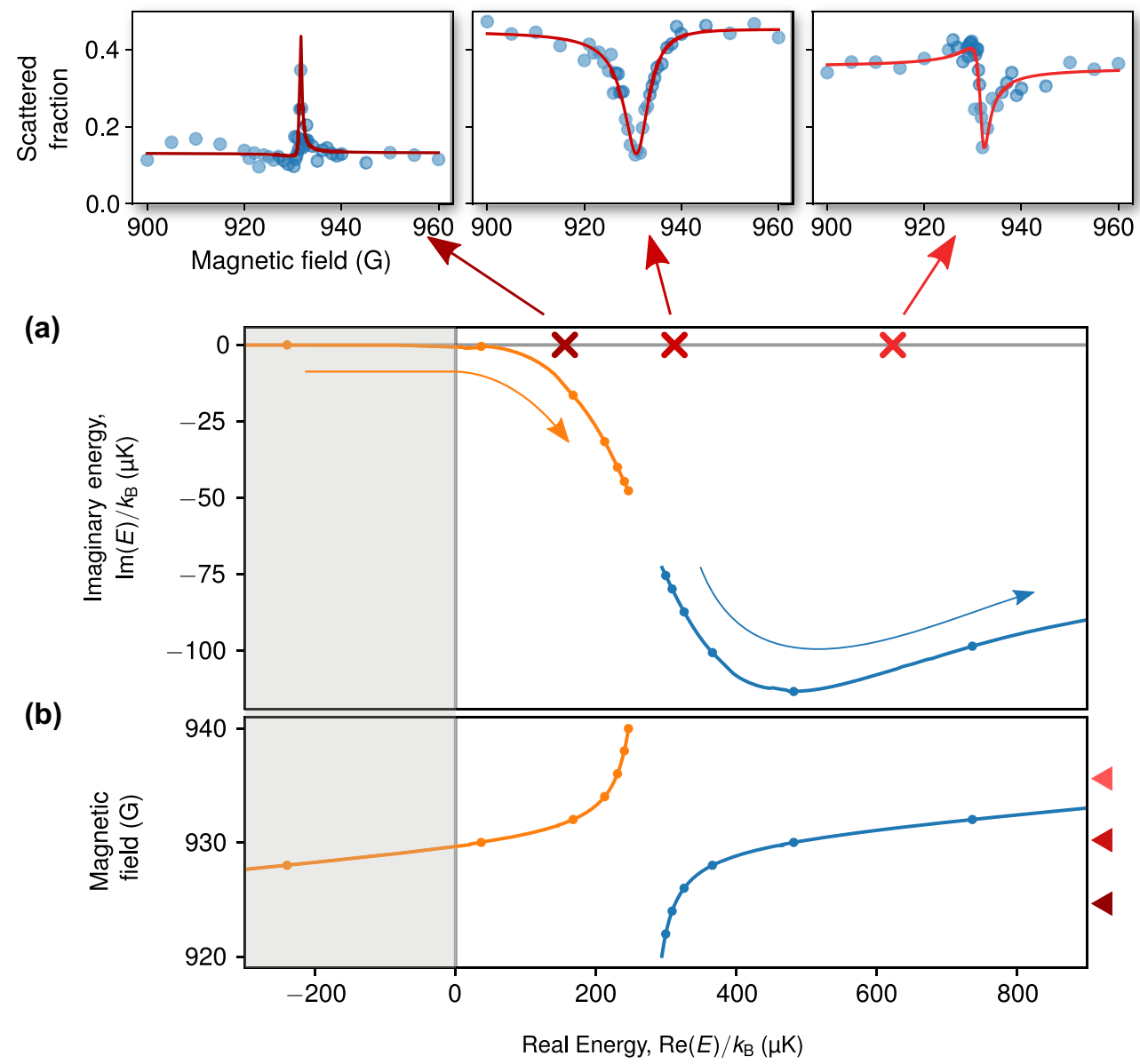

FIG. 4. (a) Rb $S$-matrix pole trajectories as functions of magnetic field with dots spaced every 2 G. The two poles can be seen to move quickly when outside of the vicinity of 200-400 $\mu \mathrm{K}$. Inset above shows the observed scattering at three fixed energies. The narrow Fano profile at $156 \mu \mathrm{K}$ is a result of the orange pole moving past quickly. At $303 \mu \mathrm{K}$, the broad Fano dip is the combined effect of the blue pole leaving, and the orange pole arriving and their intermediate interference. The resonance at $697 \mu \mathrm{K}$ is due to the pass-by of the blue pole. (b) The avoided crossing nature of the trajectories of (a) highlighted by viewing the real energy position of the poles in the magnetic field. The region below threshold is shaded grey, and the data in this region are derived from direct calculations of bound state positions in the coupled channels.

Here, the poles swap roles and avoid crossing in the complex energy plane, akin to the well-known Landau-Zener avoided crossing [14]. The avoided crossing is captured in Fig. 4(b) which shows the real energy of the poles during a magnetic field sweep. The two poles never coincide at the same real energy.

\section{QUALITATIVE TWO-LEVEL MODEL}

The essential physics of the crossing phenomenon is revealed by considering a coupled two-channel model described by the effective Hamiltonian $[14,42,43]$

$$
H=\left[\begin{array}{cc}
\varepsilon_{1}(B) & \omega \\
\omega & \varepsilon_{2}(B)
\end{array}\right],
$$

with interchannel coupling $\omega>0$ and complex bare energies $\varepsilon_{n}(B)=E_{n}(B)-\frac{i}{2} \gamma_{n}$ which we assume intersect in their real parts at $B=B_{0}$ such that $E_{1}\left(B_{0}\right)=E_{2}\left(B_{0}\right)$. The effective Hamiltonian in Eq. (3) differs from that of a closed twolevel system by introducing an imaginary component to the diagonal elements, representing a decay into the continuum and making the matrix non-Hermitian. The eigenvalues of this system, and hence the poles of the coupled $S$ matrix, are

$$
\mathcal{E}_{ \pm}=\frac{\varepsilon_{1}+\varepsilon_{2}}{2} \pm \frac{1}{2} \sqrt{\left(\varepsilon_{1}-\varepsilon_{2}\right)^{2}+4 \omega^{2}}
$$

As previously established [43], the character of the avoided crossing can be classified into three cases by the argument of the square root in Eq. (4) at $B=B_{0}$ evaluating positive, zero, or negative. This is equivalent to comparing $\omega$ to the difference in the state widths, $\left|\gamma_{1}-\gamma_{2}\right|$, as summarized in Table I.

When $\omega=\left|\gamma_{1}-\gamma_{2}\right| / 4$ (case II), the two states and the corresponding poles will coalesce exactly $\left(\mathcal{E}_{+}=\mathcal{E}_{-}\right)$at a socalled exceptional point $[44,45]$ on the complex plane for $B=B_{0}$. Unlike a closed two-level system, this case of a "pole collision" [46] displays crossing for a nonzero coupling. In contrast, for $\omega<\left|\gamma_{1}-\gamma_{2}\right| / 4$ (case I) the two poles will coincide only in real energy, with one pole moving in front of the other. Finally, for $\omega>\left|\gamma_{1}-\gamma_{2}\right| / 4$ (case III), the poles will avoid crossing in real energy, but they will coincide in imaginary energy at $B_{0}$ where they represent two equally wide resonances. In the flow of poles, one appears to displace the 
TABLE I. Classification of $S$-matrix pole interaction.

\begin{tabular}{|c|c|c|c|}
\hline & Case I & Case II & Case III \\
\hline$\left(\epsilon_{1}-\epsilon_{2}\right)^{2}+4 \omega^{2}$ & Positive & Zero & Negative \\
\hline Coupling strength $\omega$ & $<\frac{1}{4}\left|\gamma_{1}-\gamma_{2}\right|$ & $=\frac{1}{4}\left|\gamma_{1}-\gamma_{2}\right|$ & $>\frac{1}{4}\left|\gamma_{1}-\gamma_{2}\right|$ \\
\hline Crossing & $\begin{array}{c}\text { Real energy } \\
\operatorname{Re} \mathcal{E}_{+}\left(B_{0}\right)=\operatorname{Re} \mathcal{E}_{-}\left(B_{0}\right)\end{array}$ & $\begin{array}{c}\text { Poles coincide } \\
\mathcal{E}_{+}\left(B_{0}\right)=\mathcal{E}_{-}\left(B_{0}\right)\end{array}$ & $\begin{array}{c}\text { Imaginary energy } \\
\operatorname{Im} \mathcal{E}_{+}\left(B_{0}\right)=\operatorname{Im} \mathcal{E}_{-}\left(B_{0}\right)\end{array}$ \\
\hline $\begin{array}{l}\text { Pole trajectories given by } \\
\text { Eq. (4) (assuming } E_{1} \propto B \text { and } \\
E_{2}, \gamma_{1} \text {, and } \gamma_{2} \text { constant). }\end{array}$ & $\stackrel{\vec{w}{ }_{\underline{E}}}{\longrightarrow} \underset{\operatorname{Re} E}{\longrightarrow}$ & 10 & 1 \\
\hline
\end{tabular}

other-this is exactly the strongly coupled situation established in our collision experiment.

\section{SHAPE RESONANCE SHIFT}

A striking effect of the avoided crossing in our experiment is a movement of the $300 \mu \mathrm{K}$ shape resonance feature, which happens even before the "orange pole" in Fig. 4 crosses the open channel threshold. Figure 5 shows how the shape resonance moves, nearly doubling in energy as $B$ changes from 925 to $929 \mathrm{G}$. This arises through strong coupling from atomic pairs in the $d$-wave entrance channel to the closed channel molecular bound state-the origin of the Feshbach resonance-which has $d$-wave character. Interestingly, while the effect is dramatic at higher energies, the scattering behavior at threshold is not affected. This is a consequence of Wigner's threshold law [47] for partial waves with $\ell>0$, which is ultimately a statement of restricted tunneling to the inner potential region as the centrifugal barriers become wide at low energies. Meanwhile, for our system the coupling of the entrance $s$-wave channel and the $d$-wave bound state is small, so that the $s$-wave scattering at threshold is not significantly affected by the presence of the closed channel bound state.

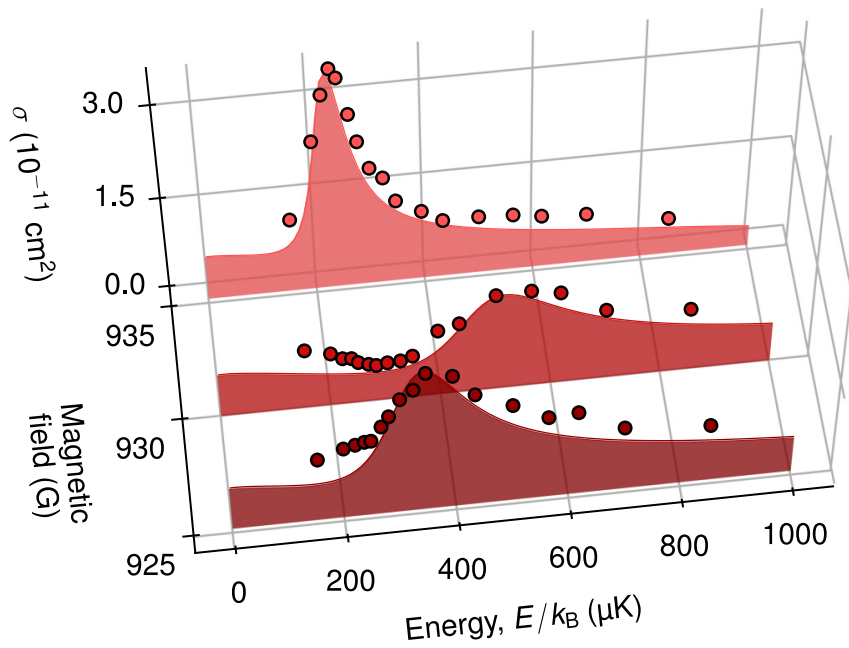

FIG. 5. Measured and calculated cross section at three magnetic fields, demonstrating the movement and restoration of the shape resonance during the avoided crossing. The color of the curves corresponds to the arrows at the right of Fig. 4(b).
Finally, Fig. 5 also shows how the shape resonance eventually reemerges at its nominal position when the magnetic field is further increased.

\section{DISCUSSION}

We have studied the interplay between two strongly interacting complex $S$-matrix poles that both reside above the open channel threshold and made use of a shape resonance and a tunable Feshbach resonance for this purpose [see Fig. 1(c)]. Our results shown in Figs. 3-5 are distinctly different from those obtained in the theory work by Dürr et al. [34], who also considered cold collisions of $|1,1\rangle$ rubidium atoms, but focused on a Feshbach resonance at $632 \mathrm{G}$. In particular, they showed that when combining this Feshbach resonance with the shape resonance, their equivalent of our Fig. 5 describes a narrow Fano profile performing a $q$ reversal as it moves across a stationary shape resonance feature. Indeed, our calculations show they examined a case I interaction (see Table I), as opposed to our case III scenario.

In a previous work [36], we explored the interplay between a magnetic Feshbach resonance and a virtual (antibound) state in the collision of potassium and rubidium. There we observed a nonmonotonic trajectory of the Feshbach resonance in a parameter space spanned by $E$ and $B$ as predicted by Ref. [48]. This result can also be interpreted in terms of interacting $S$-matrix poles. However, with the antibound state and its corresponding $S$-matrix pole residing below threshold at all times, our collider experiments, requiring $E>0$, only allowed us to see half the picture of the associated pole flow.

The experiments presented in this paper have elucidated the behavior of two strongly coupled $S$-matrix poles for colliding ${ }^{87} \mathrm{Rb}$ atoms in an $E$ - $B$-parameter space where a shape resonance and a magnetic Feshbach resonance are simultaneously present. While $S$-matrix poles constitute a well-established paradigm of quantum scattering, an experimental observation of the avoided crossing between two such poles has so far been missing. The tunability of the Feshbach resonance provides the crucial agent that allows us to record the imprint of this intriguing phenomenon on a quantum system. Curiously, in classical systems such as coupled microwave [49] and optical resonators [50], the interactions of poles and their roles in forming Fano-Feshbach resonances and exceptional points have been studied extensively, underscoring the ubiquity of these phenomena in coupled resonant systems [51-57]. 
For atomic scattering, the "collision" of $S$-matrix poles at an exceptional point [46] (case II in Table I) remains to be experimentally observed. As noted in Ref. [58], two tuning parameters are needed to achieve this in general, whereas the experiments reported in this paper only has one-the external magnetic field. In the future, the additional tuning knob needed to access an exceptional point in a collision experiment might be provided by auxiliary electric [59] or optical [60] fields.

\section{ACKNOWLEDGMENTS}

We thank J. Croft and X. Yu for critically reading our manuscript and A. Deb for useful discussions. This work was supported by the Marsden Fund of New Zealand (Contract No. UOO1923).

\section{APPENDIX A: COUPLED CHANNELS CALCULATIONS}

To compute elements of the $S$ matrix, we solve the radial Schrödinger equation for the atomic separation using numerical integration. The system is described using a coupled-channels Hamiltonian for rubidium derived from a model by Strauss et al. [61], additionally including CasimirPolder long-range interactions [62]. The Hamiltonian is experimentally determined and models the internal magnetic structure of the atom, $X^{1} \Sigma_{+}$and $a^{3} \Sigma^{+}$molecular potentials, dipole-dipole interactions, and the centrifugal barrier of the $\ell>0$ angular momentum states. The integration of the coupled equation uses the log-derivative method of Manolopoulos [63] as described in Ref. [64] and implemented in MATLAB computer code [65].

\section{APPENDIX B: ANALYTIC CONTINUATION OF $S$ MATRIX}

A function over the field of complex numbers is said to be analytic if its complex derivative is well defined. Analytic functions are subject to a uniqueness theorem which states that if two analytic functions are identical along a curve, then they are identical everywhere. The $S$ matrix is analytic and in our work it is provided on the real, positive energy axis by our coupled channels calculations (see Appendix A). In practice, analytical continuation into the complex plane can be performed by finding a so-called Padé approximant that matches the calculated $S$ matrix on the real line.

The Padé approximant [66] is a rational of two finite-order polynomials,

$$
f^{[N, M]}(z)=\frac{P(z)}{Q(z)},
$$

where $P(z)$ and $Q(z)$ are polynomials of degree $N$, and $M$ respectively, given by

$$
\begin{aligned}
& P(z)=\sum_{i=0}^{N} a_{i} z^{i}, \\
& Q(z)=\sum_{i=0}^{M} b_{i} z^{i},
\end{aligned}
$$

with $b_{0}=1$ to fix the scaling of the coefficients. Padé approximants can be considered a generalization of the Taylor polynomial to include the presence of poles.

We fit a Padé approximant $f^{[4,4]}(z)$ of fourth order in both numerator and denominator to the $S$ matrix calculated at a given magnetic field by linear least-squares methods $[4,67]$. From the fitted $f^{[4,4]}(z)$, the $S$-matrix poles are readily located by finding the roots of the polynomial $Q$.
[1] R. Eden, P. Landshoff, D. Olive, and J. Polkinghorne, The Analytic S-Matrix (Cambridge University Press, Cambridge, UK, 1966).

[2] J. R. Taylor, Scattering Theory: The Quantum Theory of Nonrelativistic Collisions (Dover, New York, 2006).

[3] A. Sitenko, Lectures in Scattering Theory (Pergamon, New York, 1971).

[4] V. I. Kukulin, V. M. Krasnopol'sky, and J. Horáček, Theory of Resonances (Kluwer, Dordrecht, 1989).

[5] S. Ceci, M. Hadžimehmedović, H. Osmanović, A. Percan, and B. Zauner, Fundamental properties of resonances, Sci. Rep. 7, 45246 (2017).

[6] P. A. Zyla et al. (Particle Data Group), Review of particle physics, Prog. Theor. Exp. Phys. 2020, 083C01 (2020).

[7] H. Nussenzveig, The poles of the $S$-matrix of a rectangular potential well of barrier, Nucl. Phys. 11, 499 (1959).

[8] R. M. Potvliege and R. Shakeshaft, Movement and interplay of the bound state, resonance, and shadow poles of the scattering amplitude in multiphoton processes, Phys. Rev. A 38, 6190 (1988).

[9] J. Dąbrowski, Poles of the $S$-matrix for a complex square well potential, J. Phys. G 23, 1539 (1997).
[10] B. Belchev, S. Neale, and M. Walton, Flow of S-matrix poles for elementary quantum potentials, Can. J. Phys. 89, 1127 (2011).

[11] A. Rácz, P. Salamon, and T. Vertse, Trajectories of $S$-matrix poles in a new finite-range potential, Phys. Rev. C 84, 037602 (2011).

[12] R. P. Meeten, G. S. Docherty-Walthew, and G. V. Morozov, Flow dynamics of the resonances of a two-dimensional circular quantum well, Phys. Rev. A 99, 042126 (2019).

[13] W. Vanroose, P. V. Leuven, F. Arickx, and J. Broeckhove, Double poles of the S-matrix in a two-channel model, J. Phys. A 30, 5543 (1997).

[14] I. Rotter, Dynamics of quantum systems, Phys. Rev. E 64, 036213 (2001).

[15] S. J. J. M. F. Kokkelmans, J. N. Milstein, M. L. Chiofalo, R. Walser, and M. J. Holland, Resonance superfluidity: Renormalization of resonance scattering theory, Phys. Rev. A 65, 053617 (2002).

[16] S. A. Rakityansky and N. Elander, Analyzing the contribution of individual resonance poles of the S-matrix to two-channel scattering, Int. J. Quantum Chem. 106, 1105 (2006).

[17] P. Kłosiewicz, W. Vanroose, and J. Broeckhove, Numerical continuation of bound and resonant states of the two-channel Schrödinger equation, Phys. Rev. A 85, 012709 (2012). 
[18] H. M. J. M. Boesten, C. C. Tsai, J. R. Gardner, D. J. Heinzen, and B. J. Verhaar, Observation of a shape resonance in the collision of two cold ${ }^{87} \mathrm{Rb}$ atoms, Phys. Rev. A 55, 636 (1997).

[19] X.-C. Yao, R. Qi, X.-P. Liu, X.-Q. Wang, Y.-X. Wang, Y.-P. Wu, H.-Z. Chen, P. Zhang, H. Zhai, Y.-A. Chen, and J.-W. Pan, Degenerate Bose gases near a $d$-wave shape resonance, Nat. Phys. 15, 570 (2019).

[20] T. Volz, S. Dürr, N. Syassen, G. Rempe, E. van Kempen, and S. Kokkelmans, Feshbach spectroscopy of a shape resonance, Phys. Rev. A 72, 010704(R) (2005).

[21] R. Legere and K. Gibble, Quantum Scattering in a Juggling Atomic Fountain, Phys. Rev. Lett. 81, 5780 (1998).

[22] N. R. Thomas, N. Kjærgaard, P. S. Julienne, and A. C. Wilson, Imaging of $s$ and $d$ Partial-Wave Interference in Quantum Scattering of Identical Bosonic Atoms, Phys. Rev. Lett. 93, 173201 (2004).

[23] C. Buggle, J. Léonard, W. von Klitzing, and J. T. M. Walraven, Interferometric Determination of the $s$ and $d$-Wave Scattering Amplitudes in ${ }^{87}$ Rb, Phys. Rev. Lett. 93, 173202 (2004).

[24] R. Thomas, K. O. Roberts, E. Tiesinga, A. C. J. Wade, P. B. Blakie, A. B. Deb, and N. Kjærgaard, Multiple scattering dynamics of fermions at an isolated p-wave resonance, Nat. Commun. 7, 12069 (2016).

[25] Ignoring weak spin-spin dipole interactions, collisions between atoms, 1 and 2, conserve $m_{F_{1}}+m_{F_{2}}=2$ as well as mechanical angular momentum $\ell$ [26]. Hence coupling of the entrance $|1,1\rangle+|1,1\rangle d$-wave channel is restricted to the channels $|1,1\rangle+|2,1\rangle,|1,0\rangle+|2,2\rangle,|2,0\rangle+|2,2\rangle$, and $|2,1\rangle+|2,1\rangle$ with $\ell=2$ [27]. The bound state that corresponds to the Feshbach resonance that our study makes use of is predominantly generated by the latter two channels.

[26] E. Tiesinga, C. J. Williams, P. S. Julienne, K. M. Jones, P. D. Lett, and W. D. Phillips, A spectroscopic determination of scattering lengths for sodium atom collisions, J. Res. Natl. Inst. Stand. Technol. 101, 505 (1996).

[27] F. H. Mies, E. Tiesinga, and P. S. Julienne, Manipulation of Feshbach resonances in ultracold atomic collisions using timedependent magnetic fields, Phys. Rev. A 61, 022721 (2000).

[28] E. A. Donley, N. R. Claussen, S. L. Cornish, J. L. Roberts, E. A. Cornell, and C. E. Wieman, Dynamics of collapsing and exploding Bose-Einstein condensates, Nature (London) 412, 295 (2001).

[29] C. A. Regal, M. Greiner, and D. S. Jin, Observation of Resonance Condensation of Fermionic Atom Pairs, Phys. Rev. Lett. 92, 040403 (2004).

[30] M. W. Zwierlein, C. A. Stan, C. H. Schunck, S. M. F. Raupach, A. J. Kerman, and W. Ketterle, Condensation of Pairs of Fermionic Atoms Near a Feshbach Resonance, Phys. Rev. Lett. 92, 120403 (2004).

[31] C. R. Cabrera, L. Tanzi, J. Sanz, B. Naylor, P. Thomas, P. Cheiney, and L. Tarruell, Quantum liquid droplets in a mixture of Bose-Einstein condensates, Science 359, 301 (2018).

[32] A. Badalyan, L. Kok, M. Polikarpov, and Y. Simonov, Resonances in coupled channels in nuclear and particle physics, Phys. Rep. 82, 31 (1982).

[33] R. E. Peierls, Complex eigenvalues in scattering theory, Proc. R. Soc. London, Ser. A 253, 16 (1959).

[34] S. Dürr, T. Volz, N. Syassen, G. Rempe, E. van Kempen, S. Kokkelmans, B. Verhaar, and H. Friedrich, Dissociation of
Feshbach molecules into different partial waves, Phys. Rev. A 72, 052707 (2005).

[35] A. Marte, T. Volz, J. Schuster, S. Dürr, G. Rempe, E. G. M. van Kempen, and B. J. Verhaar, Feshbach Resonances in Rubidium 87: Precision Measurement and Analysis, Phys. Rev. Lett. 89, 283202 (2002).

[36] R. Thomas, M. Chilcott, E. Tiesinga, A. B. Deb, and N. Kjærgaard, Observation of bound state self-interaction in a nano-eV atom collider, Nat. Commun. 9, 4895 (2018).

[37] C. S. Chisholm, R. Thomas, A. B. Deb, and N. Kjærgaard, A three-dimensional steerable optical tweezer system for ultracold atoms, Rev. Sci. Instrum. 89, 103105 (2018).

[38] B. J. Sawyer, M. Chilcott, R. Thomas, A. B. Deb, and N. Kjærgaard, Deterministic quantum state transfer of atoms in a random magnetic field, Eur. Phys. J. D 73, 160 (2019).

[39] R. Thomas and N. Kjærgaard, A digital feedback controller for stabilizing large electric currents to the ppm level for Feshbach resonance studies, Rev. Sci. Instrum. 91, 034705 (2020).

[40] H. Friedrich, Scattering Theory (Springer, Berlin, 2013).

[41] See Supplemental Material at http://link.aps.org/supplemental/ 10.1103/PhysRevResearch.3.033209 for a movie of the flowing poles and their imprint on the scattering cross section.

[42] P. von Brentano and M. Philipp, Crossing and anticrossing of energies and widths for unbound levels, Phys. Lett. B 454, 171 (1999).

[43] J. Okołowicz, M. Płoszajczak, and I. Rotter, Dynamics of quantum systems embedded in a continuum, Phys. Rep. 374, 271 (2003).

[44] T. Kato, Perturbation Theory of Linear Operators (Springer, New York, 1966).

[45] W. D. Heiss, The physics of exceptional points, J. Phys. A 45, 444016 (2012).

[46] K. McVoy, Nuclear resonance reactions and S-matrix analyticity, in Fundamentals in Nuclear Therory, edited by A. De-Shalit and C. Villi (International Atomic Energy Agency, Vienna, 1967), Chap. 8, pp. 419-499.

[47] E. P. Wigner, On the behavior of cross sections near thresholds, Phys. Rev. 73, 1002 (1948).

[48] B. Marcelis, E. G. M. van Kempen, B. J. Verhaar, and S. J. J. M. F. Kokkelmans, Feshbach resonances with large background scattering length: Interplay with open-channel resonances, Phys. Rev. A 70, 012701 (2004).

[49] E. Persson, I. Rotter, H.-J. Stöckmann, and M. Barth, Observation of Resonance Trapping in an Open Microwave Cavity, Phys. Rev. Lett. 85, 2478 (2000).

[50] X. Tu, L. Y. Mario, and T. Mei, Coupled Fano resonators, Opt. Express 18, 18820 (2010).

[51] Y. S. Joe, A. M. Satanin, and C. S. Kim, Classical analogy of Fano resonances, Phys. Scr. 74, 259 (2006).

[52] W. D. Heiss and G. Wunner, Fano-Feshbach resonances in twochannel scattering around exceptional points, Eur. Phys. J. D 68, 284 (2014).

[53] A. E. Miroshnichenko, S. Flach, and Y. S. Kivshar, Fano resonances in nanoscale structures, Rev. Mod. Phys. 82, 2257 (2010).

[54] M. F. Limonov, M. V. Rybin, A. N. Poddubny, and Y. S. Kivshar, Fano resonances in photonics, Nat. Photonics 11, 543 (2017). 
[55] Fano Resonances in Optics and Microwaves, edited by E. Kamenetskii, A. Sadreev, and A. Miroshnichenko (Springer, Cham, 2018).

[56] M.-A. Miri and A. Alù, Exceptional points in optics and photonics, Science 363, eaar7709 (2019).

[57] J. Wiersig, Prospects and fundamental limits in exceptional point-based sensing, Nat. Commun. 11, 2454 (2020).

[58] E. Hernández, A. Jáuregui, and A. Mondragón, Non-hermitian degeneracy of two unbound states, J. Phys. A 39, 10087 (2006).

[59] B. Marcelis, B. Verhaar, and S. Kokkelmans, Total Control Over Ultracold Interactions Via Electric and Magnetic Fields, Phys. Rev. Lett. 100, 153201 (2008).

[60] D. M. Bauer, M. Lettner, C. Vo, G. Rempe, and S. Dürr, Control of a magnetic Feshbach resonance with laser light, Nat. Phys. 5, 339 (2009).

[61] C. Strauss, T. Takekoshi, F. Lang, K. Winkler, R. Grimm, J. Hecker Denschlag, and E. Tiemann, Hyperfine, rotational, and vibrational structure of the $a^{3} \Sigma_{u}^{+}$state of ${ }^{87} \mathrm{Rb}_{2}$, Phys. Rev. A 82, 052514 (2010)

[62] M. Marinescu and L. You, Casimir-Polder long-range interaction potentials between alkali-metal atoms, Phys. Rev. A 59, 1936 (1999).

[63] D. E. Manolopoulos, An improved log derivative method for inelastic scattering, J. Chem. Phys. 85, 6425 (1986).

[64] R. Thomas, Cold collisions of ultracold atoms, Ph.D. thesis, Unversity of Otago, 2017.

[65] https://doi.org/10.5281/zenodo.4316008.

[66] G. A. Baker and P. Graves-Morris, Padé Approximants, 2nd ed., Encyclopedia of Mathematics and its Applications (Cambridge University Press, Cambridge, UK, 1996).

[67] P. S. Bingham and J. D. Gorfinkiel, reskit: A toolkit to determine the poles of an S-matrix, Comput. Phys. Commun. 239, 272 (2019). 